九州大学学術情報リポジトリ

Kyushu University Institutional Repository

Use of Interactive Evolutionary Computation with Simplified Modeling for Computationally Expensive Layout Design Optimization

Kamalian, Raffi R.

Faculty of Design, Kyushu University

Agogino, Alice M.

BEST Lab, University of California

Takagi, Hideyuki

Faculty of Design, Kyushu University

http://hdl. hand le. net/2324/1808438

出版情報 : IEEE Congress on Evolutionary Computation (CEC). 2007, pp.4124-4129, 2007-09. IEEE バージョン：

権利関係 : 


\title{
Use of Interactive Evolutionary Computation with Simplified Modeling for Computationally Expensive Layout Design Optimization
}

\author{
Raffi R. Kamalian, Alice M. Agogino, Hideyuki Takagi
}

\begin{abstract}
This paper presents the use of Interactive Evolutionary Computation (IEC) as a method to allow a human user to embed their expert domain knowledge and experience to overcome the deficiencies of Modified Nodal Analysis (MNA)-based EC in a fraction of the time associated with computationally expensive Finite Element Modeler (FEM)-based EC. From our comparison tests for the design of a MEMS resonating mass, results show that MNA-based IEC performance was within $10 \%$ of FEM-based EC, yet it performed in only $1 / 24^{\text {th }}$ of the computation time.
\end{abstract}

\section{INTRODUCTION}

$\mathrm{T}$ he use of Evolutionary Computation (EC) for optimization and synthesis is an emerging field in the area of machine design. One of the major challenges is the high computational cost of these EC-based tools, which rely on computer simulations to predict the performance of a machine when fabricated. Designers have a choice between highly accurate but computationally expensive simulators or more limited, less accurate simulators that are many times faster.

In this paper we show that the shortcomings of less accurate simulator-based EC can be compensated for using a human interactive technique called Interactive Evolutionary Computation (IEC). We use the design of Microelectromechanical Systems (MEMS) as an illustrative example of this approach. We will compare the speed and accuracy of solutions generated by three types of EC design synthesis: EC using expensive finite element modeling, EC using simplified simulation and EC using simplified simulation and human interaction.

\section{BACKGROUND}

\section{A. Application of EC to Engineering Design}

Evolutionary Computation has proven to be a powerful tool in the field of design and optimization [1]. The use of

Manuscript received June 15, 2007. This worked was conducted in part with support from NSF grant CCR-DES/CC-0306557.

Raffi Kamalian is on the faculty of Mechanical and Automation Engineering at the Chinese University of Hong Kong, HONG KONG (e-mail: rkamalian@gmail.com).

Alice M. Agogino is on the faculty of the Mechanical Engineering Department at the University of California, Berkeley, CA 94720, USA (e-mail: agogino@berkeley.edu).

Hideyuki Takagi is with the Faculty of Design at Kyushu University, Fukuoka 815-8540, JAPAN (e-mail: takagi@design.kyushu-u.ac.jp). genetic algorithms for layout design has also been demonstrated in a wide range of areas including electronic circuit design [2],[3], floor plan design [4], wireless sensor network layout design [5] and PC case design [6].

One benefit that EC has over conventional optimization algorithms is the fact that they do not rely on the gradient of an objective value or values. This allows their application to many complex problems, like layout design, which contain discrete variables and where the number of dimensions available in a given design is not a fixed quantity. EC can utilize a 'black box' performance rating for a given design. This black box value is usually in the form of the simulation results from modeling software.

Advances in computer simulation have opened many complex problems to the realm of optimization. But with increased complexity comes greater computational expense (in either computing power or computation time).

\section{B. EC for Computationally Expensive Problems}

A great deal of research has focused on improving the performance of EC for use on computationally expensive problems [7]. The use of fitness approximation via simplified modeling is a popular solution to long computation time per evaluation [8], but the quality of the solutions produced may be reduced with over simplification [9]. Another approach is known as evolutionary approximation, where the current generation's fitness is estimated based on previous generations [10]. Due to inaccuracies in the approximations used by both methods, a popular area of study is in methodologies that combine both expensive accurate evaluation and inexpensive approximate evaluation into a hybrid EC [11].

\section{MEMS Design}

An example of a complex computation is the simulation of MEMS. Also known as Micromachines, MEMS are electromechanical mechanisms and transducers created using IC microfabrication techniques. The most common application of MEMS technology today is in the field of inertial sensors. MEMS accelerometers and gyros have already found their way into many consumer products from automobile stability control systems to video game joysticks.

Such MEMS devices are complex in layout, containing dozens of dimensions and parameters to be tuned as well as numerous performance objectives that must be calculated. Just the mechanical simulation of such complex systems 
presents a significant computational expense. A key parameter in the case of many MEMS devices is the resonant frequency of a suspended mass. This frequency is a function of the mechanical stiffness of the suspension elements (springs) and the mass of the suspended shuttle and springs, all of which are a function of the geometry of each component as well as the material properties from which they are made. In addition to this value, the stiffness of the suspension in on-axis and off-axis directions as well as the overall area occupied by the device are key concerns. Currently the design process in research and industry involves simplified calculations and numerous rounds of trial-and-error simulation and/or prototype fabrication.

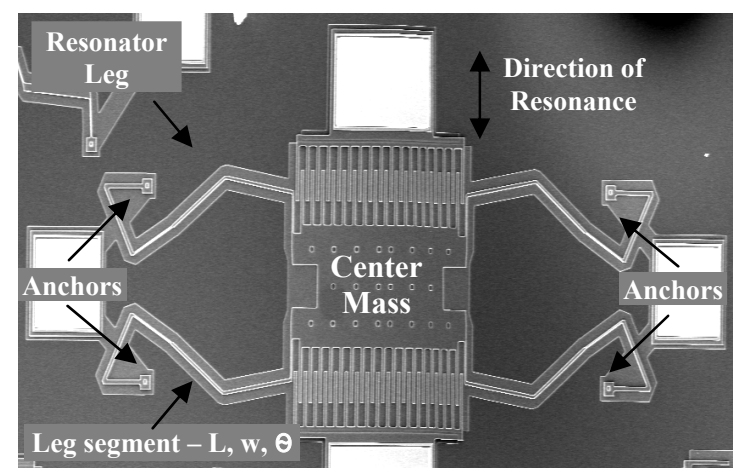

Fig. 1. Micrograph of 4- legged resonating mass test structure. The center mass is anchored to the substrate via four flexible legs. The comb drives can be used to generate motion [12].

Fig. 1 shows a scanning electron micrograph of a sample MEMS resonating structure. The center mass is suspended by four symmetric legs, each anchored to the silicon substrate at their ends. Each leg is formed by a series of straight segments, each segment with its own length, width and angle of rotation. The center mass has two electrostatic actuators known as 'comb drives' to create and sense motion relative to the substrate. By applying an $\mathrm{AC}$ voltage to a comb drive, reciprocating motion can be generated. The motion is maximum at the device's resonant frequency.

\section{Simulation of MEMS}

The most accurate tools for simulating the performance of complex MEMS devices use finite element methods (FEM), where an object is represented by a model comprised of many simplified elements, each defined by simple constitutive equations. Thus a system of simultaneous equations is generated and can be solved. FEM is an approximation; therefore the size of the elements, the element types and the boundary conditions applied affect the accuracy of the results. With the proper modeling conditions and material parameters, FEM tools have shown good accuracy in predicting the performance of fabricated MEMS structures despite uncertainties in the MEMS fabrication process [12].

A key limitation of FEM is the expense of simulation.
Depending on the type of simulation being performed and the complexity of the model, the simulation may take several seconds to several minutes to solve for the various performance objectives. This expense is bearable for simple validation, but impractical for an optimization algorithm that may require thousands or tens of thousands of design evaluations. One of the most popular FEM packages is ANSYS [13]. Because of its automated model generation scripting language and batch processing capabilities, it is well suited for use in evolutionary synthesis applications, where script files can be automatically generated for a given geometry and then evaluated via an external API.

An alternate to FEM is the use of Modified Nodal Analysis (MNA). The best known application of MNA is in the simulation of analog electronic circuits using the SPICE program [14]. In the circuits case, transistors, resistors and other circuit elements are represented by simple mathematical models which are then amassed into a large algebraic matrix. The patterns of interconnectivity (e.g. the flow of current from one element to another) are also contained in the matrix and it can be solved relatively quickly. Likewise MNA can be applied to mechanical devices. In the case of a MEMS structure, elements such as beams carry stiffness and mass (based on their physical dimensions) and they transmit motion and force. The use of MNA for MEMS simulation was developed by UC Berkeley researchers and implemented in the SUGAR tool [15]. Because it uses lumped parameter modeling, the accuracy is less than that of an FEM tool, but the simulation time can be many times faster. A key drawback however is the inability to simulate the conditions within an element, for example the internal stresses at different portions within beam. Furthermore not all complex geometries can necessarily be simulated accurately. However, if MNA simulations are limited to 'safe' regions of the design space, the simulation results for suspension stiffness and resonant frequencies are usable in a design synthesis or optimization tool [12].

\section{E. Evolutionary Synthesis of MEMS using MNA analysis}

A MEMS synthesis tool using a multiobjective genetic algorithm (MOGA) was first demonstrated by Zhou in [16]. In [12], resonating mass structures synthesized using the MNA-based MOGA were fabricated in a MEMS surface micromachining fabrication process (Fig. 2). A characterization study of the fabricated devices highlighted the ability of the MNA simulator to properly predict the performance of the design when fabricated. While the performance predictions were good for some designs, others performed poorly, and it was discovered that these designs were highly sensitive to phenomena that could not be simulated by MNA, particularly the impact of residual stress within the structure after fabrication. 


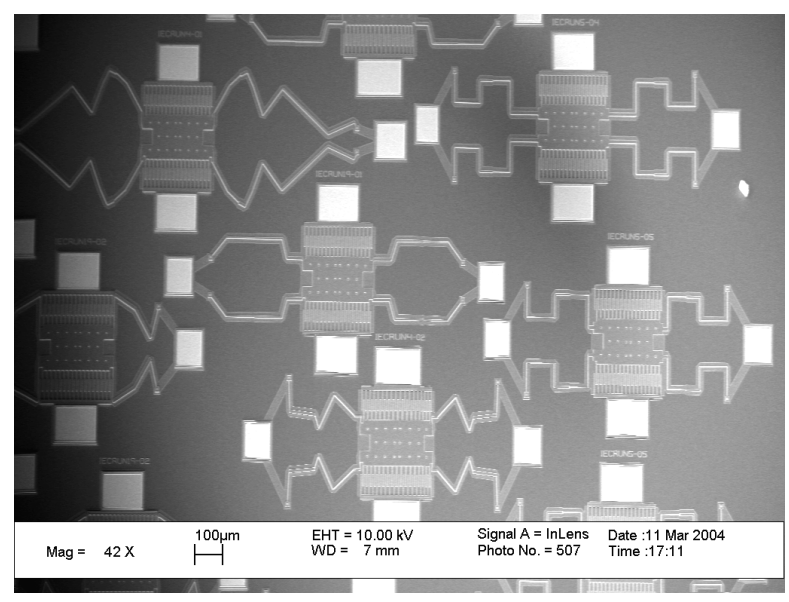

Fig. 2. Electron micrograph of various EC generated designs for MEMS resonating mass devices fabricated on a silicon wafer. Each device is approximately $1 \mathrm{~mm}$ in width.

In MEMS, residual stress is caused by the thermal expansion rate mismatch between the various materials in the MEMS structure, which is fabricated at high temperatures. After fabrication there may be residual stresses in some layers of the structure (either compressive or tensile). As the following equation shows, for a resonating fixed-fixed beam a compressive residual stress can cause a shift in the resonant frequency:

$$
\omega_{r^{\prime}}=\omega_{r} \sqrt{1-\frac{\sigma_{\text {resid }}}{\sigma_{\text {crit }}}}[17]
$$

where $\sigma_{\text {resid }}$ is the residual stress in the beam and $\sigma_{\text {crit }}$ is the critical stress in a beam at which buckling occurs:

$$
\sigma_{c r i t}=\frac{4 \pi E I}{A L^{2}}
$$

where $A$ is beam cross-sectional area, $I$ is second moment of area, $E$ is Young's Modulus for the beam and $L$ is the length of the beam. As $\sigma_{\text {resid }} / \sigma_{\text {crit }}$ gets closer to unity, the design will undergo a larger shift in resonant frequency. As these equations show, designs with longer lengths and/or smaller beam widths will have a lower critical stress and be more susceptible to frequency shift. Unfortunately the actual amount of residual stress can vary from one fabrication run to another, therefore making the actual level somewhat difficult to predict in advance.

In addition to characterizing the performance of the MNA simulator, the fabrication/characterization experiment also confirmed the capability of FEM to properly predict the performance of the fabricated designs. Unlike MNA, FEM has the ability to including the effects of residual stress on resonant frequency. Since this work was performed, properly defined FEM simulations have been used in place of fabrication to predict the performance of a given MEMS device when fabricated. Fig. 3a shows an example FEM model for a MOGA generated design; simulated using ANSYS. Fig. $3 b$ shows the first mode shape during resonance.

\section{F. FEM vs. MNA for MEMS synthesis}

The situation with these two simulation approaches leads us to a fundamental problem: FEM can simulate all the physical phenomena required to synthesize a MEMS device that will perform as predicted when fabricated, but the simulation time required makes it less practical for applications that require a high number of functional evaluations, such as required in evolutionary computation or optimal design. MNA is sufficiently accurate in some situations, is orders of magnitude faster, and has been shown to work well in EC test problems. However, because of its simplified approach, it is unable to properly simulate all the necessary physical phenomena needed to create a device that performs well when fabricated in a real world environment, where the device may be subject to uncontrollable parameters like residual stress that could negatively impact the performance of the real device.

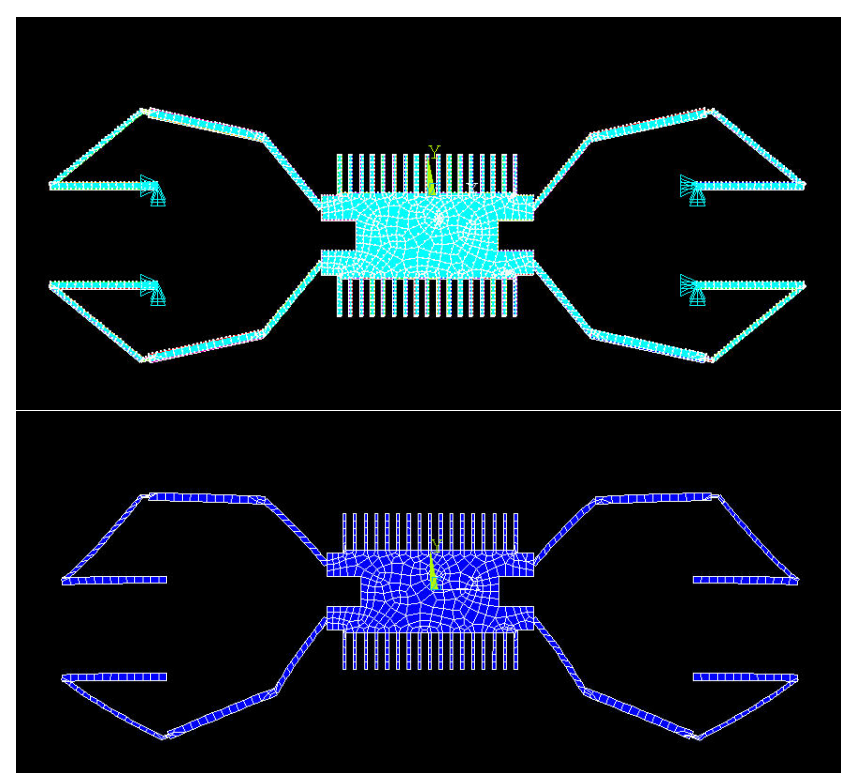

Fig. 3. Sample output from FEM simulator. (a) Finite element model of suspended portion of MEMS resonating mass device with leg anchor points represented as displacement constraints. (b) FEM generated image of first resonant frequency mode shape. Direction of resonance is in the vertical direction.

\section{INTERACTIVE EVOLUTIONARY COMPUTATION}

An alternate to conventional EC is Interactive Evolutionary Computation (IEC). Conventional EC relies on an equation or simulator to give the fitness, whereas IEC uses a human to evaluate the fitness of a design [19]. This approach has primarily been used for aesthetic or sensoryperception related design, but has also been adapted to engineering design [20]. This allows the human designer a degree of interaction with the evolution process. It has been suggested that this interactivity allows the human designer 
to use their engineering experience, expert domain knowledge, qualitative judgment and human visual perception to influence the direction of the evolutionary search.

In the case of the MEMS resonator design problem, the MNA-based simulator may not be able to properly account for issues such as residual stress in the structure, but a human designer can predict which designs are more susceptible to these problems and via IEC avoid them.

Several variations of IEC for engineering design have been developed, including an absolute human score version [20] and a Pareto rank-shift human score version [21]. The major difference between the two implementations is the amount of human interaction involved. The interactivity required is important for IEC because unlike conventional automated EC where the evaluation engine is a tireless computer, IEC's main limitation is fatigue in the human user. The rank-shift IEC implementation was found to be the less taxing of the two methods on human users.

The Pareto rank-shift method allows the human to interact as much or as little as desired by promoting or demoting a design if they have a strong opinion about it or leaving the Pareto rank unchanged if they do not. Another benefit is that the human's interaction is not needed at every generation; they can participate periodically, acting more in a supervisory role (Fig. 4). This approach is similar to the generation-based evolution control approaches discussed in [7] for use in computationally expensive problems, where we alternate between automated evaluation and human evaluation.

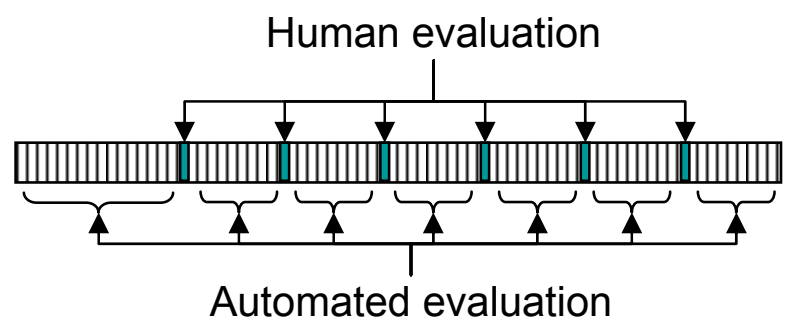

Fig. 4. Schematic of interspersed human interaction in automated evolutionary synthesis

\section{Methodology}

Our goal in this paper is to perform a comparison test to show that using IEC can improve performance over a noninteractive MNA-based EC tool sufficiently to make it a viable (more practical) alternative to using FEM based EC. In order to do this we need to perform testing to support two hypotheses:
1) Human interaction (IEC) generated designs perform better than MNA-based EC generated designs and perform as well, or close to the level of FEM-based EC generated designs.

2) The speed of MNA-based IEC is significantly faster than FEM-based EC.

For part 1, the comparison of performance of the generated designs was made using the methodology and user test data from [21]. The top two designs from each test run of the MNA-based synthesis programs are selected and the sensitivity of each design to residual stress is calculated (using FEM-based simulation). The sensitivity is defined by the resonance frequency shift caused by residual stress (less sensitivity $=$ smaller shift $=$ better design).

These results were then analyzed using the analysis of variance (ANOVA) test to verify a statistically significant difference in performance between the different EC/IEC output.

For part 2, the comparison was relatively straightforward: The average time to perform an evolution of equal population size and number of generations was compared between MNA-based IEC and FEM-based EC.

We do not expect that the human assisted EC to outperform an FEM-based EC, which can explicitly calculate factors that a human can only make an educated guess about, but if MNA-based IEC can perform close to FEM-based EC performance in a fraction of the time, we feel this would be sufficient evidence that this method is a viable compromise.

We used the design of a four legged resonating mass MEMS device as the test problem. We used our MOGA synthesis program with four objective goals: resonant frequency $\left(\omega_{\mathrm{r}}\right)$, lateral stiffness $\left(\mathrm{K}_{\text {lateral }}\right)$, vertical stiffness $\left(\mathrm{K}_{\text {vertical }}\right)$, and Area. (Table I). As the fourth objective has a goal of zero area, which is impossible to physically achieve, it is impossible to synthesize a design that meets all four goals. Our primary interest was to set the goals such that they would achieve the desired value of $\omega_{\mathrm{r}}$, with a minimal area. $\mathrm{K}_{\text {lateral }}$ and $\mathrm{K}_{\mathrm{vertical}}$ were intended not as fixed goals, but to ensure that the suspension generated would be less stiff in the vertical direction so that resonance in the lowest natural frequency was also in that direction

TABLE I: OBJECTIVES AND GOAL VALUES FOR EC FOR MEMS RESONATING MASS SYNTHESIS

\begin{tabular}{|l|l|}
\hline Objective: & Goal \\
\hline Resonant frequency $\left(\omega_{\mathrm{r}}\right)$ & $10,000 \mathrm{~Hz}$ \\
\hline $\mathrm{K}_{\text {lateral }}$ & $100 \mathrm{~N} / \mathrm{m}$ \\
\hline $\mathrm{K}_{\text {vertical }}$ & $0.5 \mathrm{~N} / \mathrm{m}$ \\
\hline Area & Minimize \\
\hline
\end{tabular}

Building on the previous work presented in [21] for purposes of comparison, an evolution of 80 generations with a population of 80 individuals was chosen as a sufficiently large evolution size to demonstrate the capabilities of IEC 
using MNA evaluation (Table II).

TABLE II: SETTINGS FOR RANK-SHIFT IEC USER TEST

\begin{tabular}{|l|l|}
\hline Property & Setting \\
\hline Population Size & 80 \\
\hline Generations & 80 \\
\hline $\begin{array}{l}\text { Interval for } \\
\text { human interaction }\end{array}$ & $\begin{array}{l}\text { Every } 10^{\text {th }} \\
\text { generations }\end{array}$ \\
\hline $\begin{array}{l}\text { Starting point for } \\
\text { human interaction }\end{array}$ & $20^{\text {th }}$ generation \\
\hline $\begin{array}{l}\text { Total number of } \\
\text { human interaction } \\
\text { generations }\end{array}$ & $\begin{array}{l}6 \\
\left(20,30,40,50,60,70^{\text {th }}\right. \\
\text { generations })\end{array}$ \\
\hline
\end{tabular}

For the FEM based evolution, a modified set of objectives was chosen. Because FEM has the ability to include internal effects into the beam elements, a resonant frequency under residual stress can be also calculated. As FEM can calculate this shift explicitly, and ultimately it is what we want to minimize, an alternate set of objectives and goal values was chosen: resonant frequency $\left(\omega_{\mathrm{r}}\right)$, resonant frequency under residual stress (a compressive stress of $5 \mathrm{MPa}$ is chosen as an arbitrary amount for the sake of the simulation), the suspension stiffness ratio $\left(\mathrm{K}_{\text {lateral }} / \mathrm{K}_{\text {vertical }}\right)$, and Area. These objectives, while somewhat different than the MNA-based EC objectives should produce designs that match our ultimate goal - resonators with a resonant frequency close to $10 \mathrm{kHz}$ that are insensitive to residual stress, have a high stiffness ratio in the proper direction and are of a restrained area. The objectives and the goal amounts used by the FEMbased EC can be seen in Table III.

TABLE III: OBJECTIVES AND GOAL VALUES FOR EC FOR MEMS
\begin{tabular}{|l|l|}
\hline RESONATING MASS SYNTHESIS \\
\hline Objective: & Goal \\
\hline Resonant frequency $\left(\omega_{\mathrm{r}}\right)$ & $10,000 \mathrm{~Hz}$ \\
\hline $\begin{array}{l}\text { Resonant frequency with } \\
5 \mathrm{MPa} \text { compressive } \\
\text { residual stress }\end{array}$ & $10,000 \mathrm{~Hz}$ \\
\hline $\mathrm{K}_{\text {lateral }} / \mathrm{K}_{\text {vertical }}$ & \\
\hline Area & 200 \\
\hline
\end{tabular}

In order to create a baseline for comparison, the FEM EC, using the FEM package ANSYS, is also run for a population size of 80 over 80 generations. It is difficult to create an identical comparison, as the two modeler's EC objectives are not completely the same and the fact that IEC performance varies from user to user [21]. Also the high computational expense of the FEM evaluation limits the total number of test cases available for comparison - 10 test runs were used for this experiment.

All tests were run on a single PC for comparison. The system has a $3.4 \mathrm{GHz}$ Pentium 4 processor with $1 \mathrm{~Gb}$ of RAM.

\section{RESULTS}

\section{A. Quality of Output}

Table IV shows the results of our comparison tests. MNA-based EC output had an average resonant frequency shift (sensitivity to residual stress) as high as $73 \%$, whereas human intervention (MNA-based IEC) reduced that to only $58 \%$, this compares favorably to FEM-based EC, which was at $48 \%$.

Using the ANOVA single value test, these groups can be said to be significantly different with $98 \%$ confidence. This result is not very illuminating except that it shows that while MNA-based IEC is not at the level of FEM-based EC, it is significantly better than the non-interactive MNA-based EC.

TABLE IV: COMPARISON OF RESULTS OF MNA-BASED EC, MNABASED IEC AND FEM-BASED EC SYNTHESIS TOOLS FOR 4-OBJECTIVE RESONATING MASS SYNTHESIS TEST PROBLEM.

\begin{tabular}{|c|c|c|c|}
\hline & $\begin{array}{l}\text { MNA-based } \\
\text { EC } \\
\text { (20 designs) }\end{array}$ & $\begin{array}{l}\text { MNA-based } \\
\text { Rank-shift } \\
\text { IEC } \\
\text { (26 designs) }\end{array}$ & $\begin{array}{l}\text { FEM- } \\
\text { based EC } \\
(20 \\
\text { designs })\end{array}$ \\
\hline \multicolumn{4}{|c|}{ Percent Shift in Performance with Residual Stress: } \\
\hline Average & $73 \%$ & $58 \%$ & $48 \%$ \\
\hline Std. dev & $23 \%$ & $15 \%$ & $12 \%$ \\
\hline \multirow{2}{*}{$\begin{array}{l}\text { ANOVA P- } \\
\text { value }\end{array}$} & \multicolumn{2}{|c|}{$\mathrm{P}=0.014(98 \%$ significance $)$} & \\
\hline & & \multicolumn{2}{|c|}{$\begin{array}{c}\mathrm{P}=0.016(98 \% \\
\text { significance })\end{array}$} \\
\hline \multicolumn{4}{|c|}{ Run time (Population $=80$, Generations $=80)$ : } \\
\hline Average & $\begin{array}{c}14 \text { minutes } \\
(0.23 \text { hours })\end{array}$ & $\begin{array}{c}45 \text { minutes } \\
(0.75 \text { hours })\end{array}$ & $\begin{array}{c}1075 \\
\text { minutes } \\
(17.9 \\
\text { hours }) \\
\end{array}$ \\
\hline $\begin{array}{l}\text { Speed ratio } \\
\text { compared } \\
\text { to IEC: }\end{array}$ & 0.31 & 1 & 23.9 \\
\hline
\end{tabular}

\section{B. Evolution Time}

The most dramatic difference in performance can be seen in the run times also displayed in Table IV. MNA-based EC required on average 14 minutes to perform an 80 population synthesis over 80 generations, including human interaction extended that to an average of 45 minutes, whereas FEMbased EC required 17.9 hours. Human intervention was about 3x slower than an automated MNA-based EC, but nearly $24 x$ faster than FEM-based EC.

An additional complication is the fact that our FEM modeler, ANSYS crashes on occasion, requiring a human to terminate the program manually. While the EC program can skip this design and continue with the evolution with little detriment, the average run time of 17.9 hours occurs only if an operator is on hand to intervene in the case of a crash (in other words even the 'automatic' alternative still requires human assistance from time to time). Without this human 
monitoring, the overall time to generate output can be significantly longer.

\section{Discussion}

In the case of MEMS layout design, the results of our synthesis comparison tests show that using IEC with a simplified modeler can at least approach the performance level of EC using a much more complex modeler. The key difference being that the simplified modeler is still many times faster, even including the relative 'slowness' of human evaluation compared to modern microprocessors. Depending on the application, this human intervention with a simplified simulator may be able to produce output near the quality of the slower, computationally expensive simulator, in a fraction of the time. Of course, once the design structure has been optimized with MNA-based IEC and a small subset of the Pareto optimal set is selected, full finite element analysis might be applied at the end to fine-tune the final design.

\section{CONCLUSIONS/Future WORK}

In this paper we have used the layout design problem of MEMS synthesis to demonstrate that an EC using human interaction to insert expert domain knowledge, experience and human qualitative judgment to augment the output produced by a simplified simulator. This boost in performance can make up the lack of simulation capability found in more complex modelers like FEM. IEC with simplified simulation can produce results that are nearly as good as the complex simulator at a fraction of the computation time.

As this is only one case in the area of layout design, one can not draw parallels to all design domains. Additionally the same modeling options are not available for all domains.

We would like to build upon the work presented here to investigate alternate ways of increasing the capability of designers to mitigate the expenses in computationally expensive design areas. One possibility is to create a hybrid EC that uses simplified modelers and human interactivity in conjunction with FEM. This approach shadows the work already performed in the research community combining expensive computation with approximate models for optimization [8], but with the added dimension of adding human expert evaluation to augment simplified modeling.

There are many examples that could build on the rankbased IEC used in this paper, the human interaction only occurs one out of 10 generations (Fig. 3), as a periodic check to reject (or promote) designs that the human feels deserve attention. Likewise FEM could be periodically used to screen the high ranking output to confirm whether they also perform well in objectives that the MNA modeler can not evaluate. This would serve to back-up the human's expert opinion and compensate for factors they may overlook or improperly assess.

\section{REFERENCES}

[1] D. E. Goldberg, Genetic Algorithms in Search, Optimization and Machine Learning, Addison-Wesley Longman Publishing Co., Inc., Boston, MA, 1989.

[2] J. R. Koza, F. H. Bennett III, D. Andre, and M. A. Keane, "Synthesis of topology and sizing of analog electrical circuits by means of genetic programming," Computer Methods in Applied Mechanics and Engineering. vol. 186, no. 2-4, pp. 459-482, June 9, 2000.

[3] K. Deb, P. Jain, N. Gupta, and H. Maji. "Multi-objective placement of VLSI components using evolutionary algorithms." IEEE Trans. on Components and Packaging Technologies, vol. 27, no. 3, 2004.

[4] J. P. Cohoon, S. U. Hedge, W. N. Martin, and D. S. Richards, "Distributed genetic algorithms for floorplan design problem," IEEE Trans. on CAD, vol.10, no.4, pp.483-492, 1991.

[5] D. B. Jourdan and O. L. de Weck, "Layout optimization for a wireless sensor network using a multi-objective genetic algorithm," Proc. IEEE Vehicular Technology Conference, Milan, May 2004.

[6] S. Takeuchi and K, Saitou, "Design for product-embedded disassembly", Proc. of IDETC/CIE 2005, Long Beach, CA, Sept. 2005

[7] Y. Jin, "A comprehensive survey of fitness approximation in evolutionary computation," Soft Computing Journal, vol 9, pp. 3-12, 2005.

[8] Y. S. Ong, P. B. Nair, and A. J. Keane, "Evolutionary optimization of computationally expensive problems via surrogate modeling," American Institute of Aeronautics and Astronautics Journal, vol. 41, no. 4, pp. 687-696, 2003.

[9] Y. S. Ong, Z. Zhou, and D. Lim, "Curse and blessing of uncertainty in evolutionary algorithm using approximation," Proc. The 2006 IEEE World Congress on Computational Intelligence (WCCI2006), Vancouver, pp. 2928-2935, July 2006.

[10] M. Emmerich, A. Giotis, M. Ozdemir, T. Back, K. Giannakoglou: "Metamodel-assisted evolution strategies" Parallel Problem Solving from Nature, Springer-Verlag pp. 361-370, 2002.

[11] Z. Z. Zhou, Y. S. Ong, P. B. Nair, A. J. Keane and K. Y. Lum, "Combining Global and Local Surrogate Models to Accelerate Evolutionary Optimization", IEEE Transactions On Systems, Man and Cybernetics - Part C, Vol. 37, No. 1, pp. 66-76, 2007.

[12] R. Kamalian, Y. Zhang, and A.M. Agogino, "Microfabrication and characterization of evolutionary MEMS resonators," 2005 IEEE Int. Symposium on Micro-NanoMechatronics and Human Science, Nagoya, Japan, pp. 109-114, Nov. 2005.

[13] http://www.ansys.com

[14] http://bwrc.eecs.berkeley.edu/Classes/IcBook/SPICE/

[15] http://www-bsac.eecs.berkeley.edu/cadtools/sugar/sugar/

[16] N. Zhou, B. Zhu, A. M. Agogino, and K. S. J. Pister, "Evolutionary synthesis of MEMS MicroElectronicMechanical Systems design," Proc. of the Artificial Neural Networks in Engineering (ANNIE2001), pp.197-202, 2001.

[17] A. E. Galef, "Bending Frequencies of Compressed Beams", $J$. Acoustic Society of America, 44, 1968.

[18] E.P. Popov, Engineering Mechanics of Solids, Prentice Hall, New Jersey, 1990.

[19] H. Takagi, "Interactive Evolutionary Computation: Fusion of the capacities of EC optimization and human evaluation", Proceedings of the IEEE, vol. 89, no. 9, pp. 1275-1296, 2001.

[20] R. Kamalian, H. Takagi, and A.M. Agogino, "Optimized design of MEMS by evolutionary multi-objective optimization with Interactive Evolutionary Computation", Genetic and Evolutionary Computation Conference (GECCO2004), pp. 1030-1041, 2004.

[21] R. Kamalian, Y. Zhang, A.M. Agogino, and H. Takagi, "Evolutionary synthesis of micromachines using supervisory multiobjective interactive evolutionary computation", Lecture Notes in Artificial Intelligence 3930: Advances in Machine Learning and Cybernetics, Springer Verlag, Berlin, , pp. 428-437, 2006. 